Research, Society and Development, v. 9, n. 10, e7589108414, 2020

(CC BY 4.0) | ISSN 2525-3409 | DOI: http://dx.doi.org/10.33448/rsd-v9i10.8414

\title{
Correção de assimetria facial em indivíduo adulto com disjunção palatal unilateral assistida por miniparafusos (MARPE) - Nova Técnica: Relato de caso \\ Correction of facial asymmetry in an adult with unilateral palatal disjunction assisted by mini screws (MARPE) - New Technique: Case report \\ Corrección de asimetría facial en un adulto con disyunción palatina unilateral asistida por minitornillos (MARPE) - Nueva Técnica: Reporte de caso
}

Recebido: 29/09/2020 | Revisado: 01/10/2020 | Aceito: 16/10/2020 | Publicado: 18/10/2020

Edson Homem Neto

ORCID: https://orcid.org/0000-0002-6217-6699

Universidade Paranaense - Unipar, Brasil

E-mail: edsonhomemneto@gmail.com

\section{Resumo}

Introdução: Um dos primeiros problemas a serem tratados nas desordens oclusais, é a correção transversal que pode envolver uma mordida de topo, cruzada uni ou bilateral. Essas atrésias, muitas vezes, podem contribuir nas correções estéticas do sorriso, incluindo assimetrias da face. Objetivo: Visando à correção das assimetrias faciais com a expansão rápida da maxila apoiada em miniparafusos, este trabalho demonstra uma nova técnica por meio da fragilização e expansão unilateral da maxila que facilita o retorno da mandíbula deslocada para o lado ipsilateral da expansão. Relato de caso: Com a utilização de corticoperfurações realizadas na rafe palatina, como já sugerido na literatura, e complementadas também na região de crista infrazigomática no lado oposto à assimetria facial, isso favoreceu o deslocamento mandibular para o lado da expansão, corrigindo assim a estética da face. Considerações Finais: A presente abordagem de fragilização da crista infrazigomática, com o intuito de maior deslocamento de uma hemimaxila para o lado oposto ao desvio facial, pode facilitar algumas correções não cirúrgicas de indivíduos assimétricos.

Palavras-chave: Expansão maxilar; Disjunção palatal; Assimetria facial.

\footnotetext{
Abstract

One of the first problems to be treated in occlusal disorders is cross-sectional correction, which may involve a top bite, a single or bilateral. These atresias can often contribute to the aesthetic corrections of the smile including asymmetries of the face. Objective: Aiming at
} 
correcting facial asymmetries with rapid maxillary expansion supported by mini-screws, this work demonstrates a new technique through weakening and unilateral maxilla expansion that facilitates the return of the displaced mandible to the ipsilateral side of the expansion. Case report: With the use of holes made along the palate raphe, as already suggested in the literature, and also complemented in the region of the infra-zygomatic crest on the opposite side of the facial asymmetry, this favored the mandibular displacement towards the expansion side, thus correcting aesthetics of the face. Final Considerations: This approach to weakening the infra-zygomatic ridge with the intention of greater displacement of a hemi-maxilla to the opposite side to the facial deviation, may facilitate some non-surgical corrections of asymmetric individuals.

Keywords: Maxillary expansion; Palatal disjunction; Facial asymmetry.

\section{Resumen}

Introducción: Uno de los primeros problemas a tratar en los trastornos oclusales es la corrección transversal, que puede implicar una mordida superior, una mordida cruzada simple o bilateral. Estas atresias a menudo pueden contribuir a las correcciones estéticas de la sonrisa, incluidas las asimetrías del rostro. Objetivo: Con el objetivo de corregir las asimetrías faciales con rápida expansión maxilar apoyada por minitornillos, este trabajo demuestra una nueva técnica a través del debilitamiento y expansión maxilar unilateral que facilita el retorno de la mandíbula desplazada al lado ipsilateral de la expansión. Caso clínico: Con el uso de corticoperforaciones realizadas en el rafe palatino como ya se sugiere en la literatura y también complementadas en la región de la cresta infra-cigomática en el lado opuesto a la asimetría facial, esto favoreció el desplazamiento mandibular hacia el lado de expansión, corrigiendo así la cara. Consideraciones finales: Este nuevo enfoque para debilitar la cresta infra-cigomática con la intención de un mayor desplazamiento de un hemi-maxilar hacia el lado opuesto a la desviación facial, puede facilitar algunas correcciones no quirúrgicas de individuos asimétricos.

Palabras clave: Expansión maxilar; Disyunción palatina; Asimetría facial.

\section{Introdução}

As correções assimétricas da face em indivíduos adultos, muitas vezes são deixadas em um segundo plano quando as cirurgias ortognáticas não podem ser realizadas por algum motivo. O crescimento desigual dos hemiarcos estabelecidos quando da presença de 
interferências oclusais ou problemas esqueléticos não corrigidos durante a fase de crescimento, faz com que as intervenções ortopédicas e ortodônticas nem sempre consigam mascarar esses desvios, sendo o tratamento focado quase que exclusivamente na organização dos encaixes dentários de forma compensatória. Arnett \& Bergman (1993)

Com o surgimento de técnicas de disjunção palatal sem a necessidade da cirurgia assistida por estarem reforçadas com o apoio de miniparafusos, grande número de problemas transversais pôde ser corrigido com o auxílio desses dispositivos expansores com ancoragem diferenciada. Araújo et al. (2017)

Um dos grandes problemas encontrados nas disjunções são as faltas de elementos dentais que possam ajudar na ancoragem do expansor, bem como as perdas ósseas e reabsorções radiculares encontradas no início do tratamento, debilitando o periodonto de suporte do indivíduo. Mesmo sem parte de todos os problemas mencionados, os expansores convencionais acabam gerando reabsorções radiculares aos dentes de ancoragem. Garib (2006)

As disjunções palatais, que em indivíduos com idade avançada se mostravam arriscadas, podendo comprometer não só tecidos moles (necrose), mas também ósseo e radicular (fenestrações) Capelozza \& Silva Filho (1999), com o auxílio dos expansores tipo MARPE (Expansor Palatal Rápido Ancorados em Miniparafusos) trouxeram de volta aos consultórios muitos daqueles que anteriormente se negavam a fazer uma cirurgia ortognática invasiva.

Esse tipo de aparelho proporciona ao ortodontista uma nova ferramenta nas correções transversais severas, pois dispensa a necessidade de cortes cirúrgicos extensos (Le fort I) Fonseca (2015), que com o auxílio de corticoperfurações na Rafe palatina e agora também em região de Crista Infrazigomática, podem obter o mesmo resultado antes conseguido apenas com as cirurgias.

Dessa forma, este trabalho tem como objetivo, descrever uma nova técnica que utilizando-se das corticoperfurações, favorece o deslocamento unilateral da maxila que ajuda na correção das assimetrias faciais.

\section{Metodologia}

O presente trabalho mostra uma nova alternativa para as correções das assimetrias faciais, bem como uma forma de facilitar a protração maxilar quando necessária. Com a utilização de corticoperfurações realizadas sobre a rafe palatina, como já descrito por vários 
autores Lee (2010); Perim (2016); Suzuki et al. (2019); Santos et al. (2019), uma nova alternativa foi acrescentada também na região da crista infrazigomática, assim podendo ser expandido em maior quantidade apenas uma hemimaxila do lado oposto ao desvio. Trata-se de um estudo descritivo detalhado e com imagens, retrospectivo, feito por meio da técnica de observação direta. (Pereira et al., 2018), sendo uma das formas mais básicas e tradicionais de estudo clínico das áreas médicas. Os dados da paciente foram coletados em seus atendimentos por meio de anamnese e exames solicitados. Seguindo os princípios éticos, a paciente consentiu com a divulgação dos dados e exibição de imagens de seu caso, com finalidade acadêmica, por meio da assinatura de um Termo de Consentimento Livre e Esclarecido.

\section{Resultados e Discussão}

\section{Relato de caso}

Indivíduo do gênero feminino, 30,6 anos, caucasiana, mesofacial, apresentando ausência de apinhamentos ou segundos molares vestibularizados, o que representa um diagnóstico favorável para bioadaptações transversais com a utilização de aparelho autoligável, atresia maxilar e assimetria de face com desvio do mento para direita, desvio de linha média inferior de $2,5 \mathrm{~mm}$ também para a direita, mostrando o lado preferência mastigatória e de menor dimensão vertical. Da mesma forma pode ser observado o menor desenvolvimento transversal da maxila para o mesmo lado como demostrado por Planas em 1964 nas Leis da mínima dimensão vertical.

Tendo como queixa principal não só os problemas de ordem oclusais, apresentava mordida cruzada unilateral direita e abfrações nos dentes pré-molares em ambos os lados, e também dores localizadas na região de ATM (Articulação Temporomandibular) esquerda. 
(CC BY 4.0) | ISSN 2525-3409 | DOI: http://dx.doi.org/10.33448/rsd-v9i10.8414

Figuras 1 e 2. Fotos frontal em repouso e frontal do sorriso - onde podemos observar o deslocamento do mento para a direita, acompanhando o lado da mordida cruzada bem como no sorriso a menor área de corredor bucal para o mesmo lado.
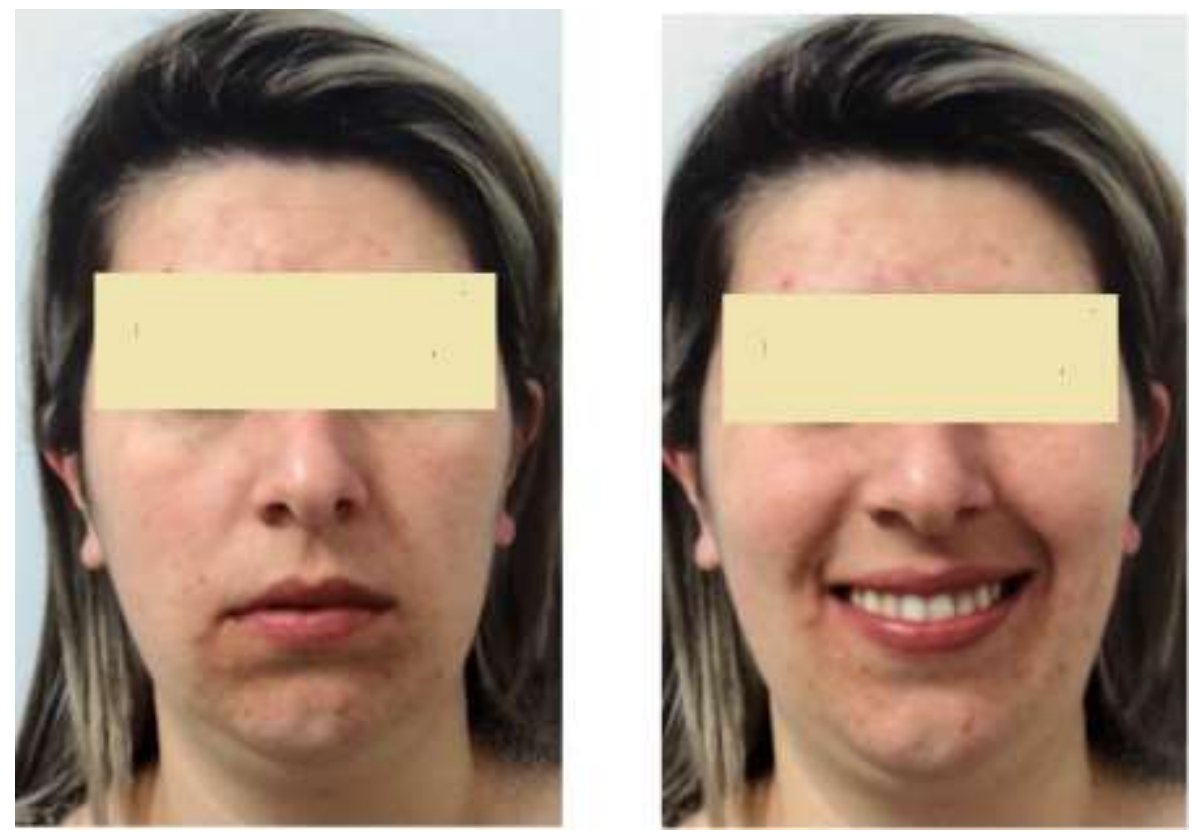

Fonte: O próprio autor.

Figuras 3. 4 e 5. Fotos intra bucal direita, frontal e esquerda. As imagens evidenciam o desvio lateral da mandíbula para a direita, resultado da mordida cruzada do mesmo lado bem como as lesões cervicais já presente na região posterior, resultado dos traumas oclusais presentes.
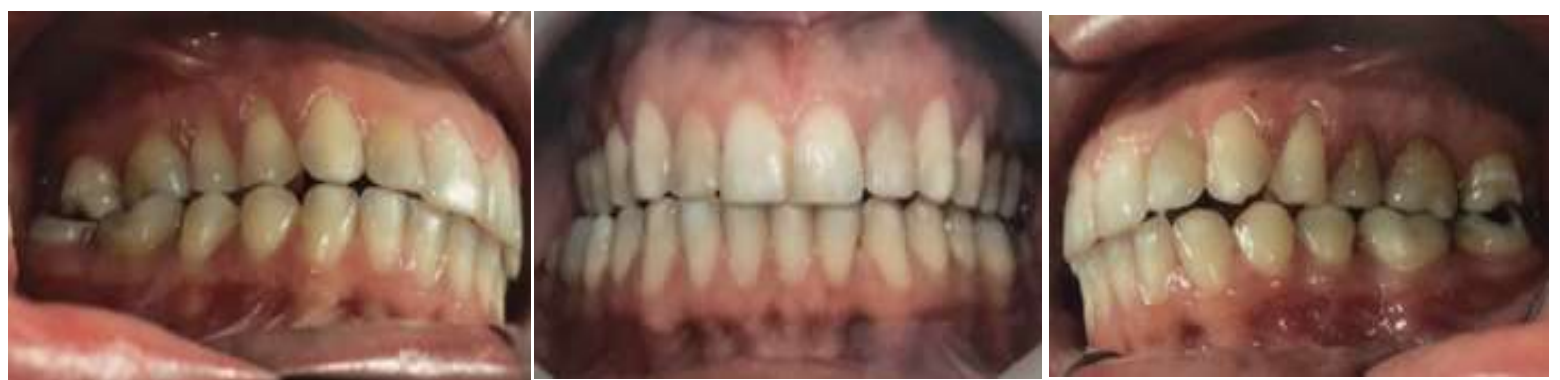

Fonte: O próprio autor.

Foi planejado como tratamento corretivo inicial a disjunção palatal com o dispositivo MARPE (Expansor Palatal Rápido Ancorado em Miniparafusos) devido à idade e a necessidade da correção do problema transversal. De uma forma diferente das propostas até então, foi planejado não só a corticoperfurações em rafe palatina, mas também na crista 
infrazigomática do lado oposto ao desvio com o intuito de trazer para este lado a mandíbula desviada e, consequentemente, não só corrigir a linha média, como também acertar a oclusão cruzada do lado direito.

Figura 6. Osso Malar ou Zigomático (evidenciado por seta a crista infra zigomática) pode ser observado a área a baixo da região do osso zigomático e acima das coroas dos dentes posteriores onde normalmente é realizado as corticoperfurações uni ou bilateral para fragilizar a resistência ao deslocamento da maxila.

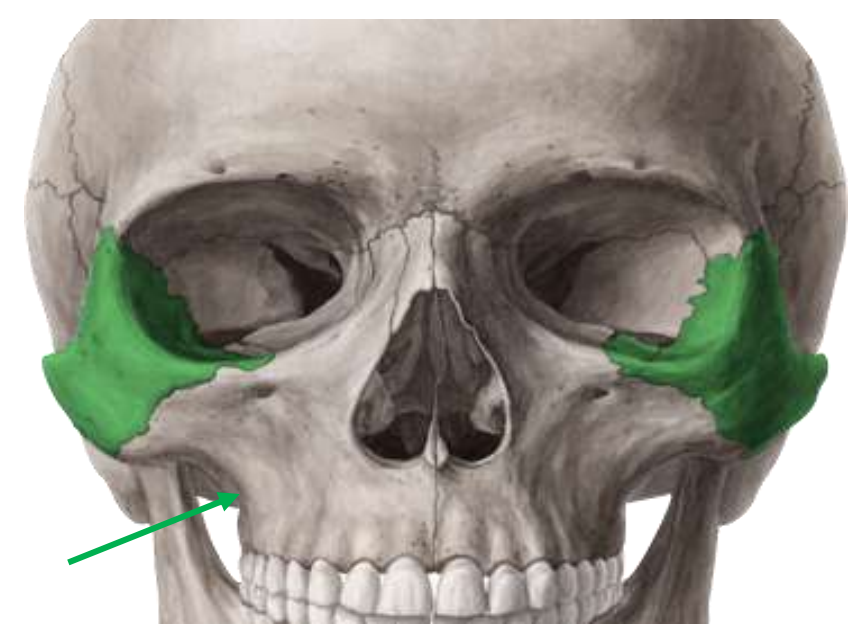

Fonte: https://www.kenhub.com/pt/library/anatomia/osso-zigomatico.

Apesar do MARPE ser um expansor apoiado em miniparafusos que são fixados lateralmente à rafe palatina, inicialmente é preconizado corticoperfurações na região de maior resistência de disjunção, sendo esta entre a 2 brida lateral do palato de posterior para anterior até a distal de primeiros molares sobre a região medial do palato. Murata et al. (2107)

Quando é necessária uma disjunção bilateral, nem sempre são realizadas fragilizações dos pilares zigomáticos Fonseca (2015) a menos que no planejamento a protração maxilar esteja como um dos procedimentos para obtenção de correções anteroposterior (mordida cruzada anterior) e uma melhora facial por comprometimento do desenvolvimento da face média, o que neste caso, favorece a maior desorganização da sutura mediana e diminui a resistência à protração da maxila.

O procedimento de expansão rápida da maxila (ERM) tem como principal finalidade a correção da atresia do arco dentário pela abertura da sutura intermaxilar e consequente incremento transversal da base óssea superior. Garib (2007)

Considerando a necessidade de mesmo antes das cimentações do aparelho MARPE já serem feitas as corticoperfurações ao longo da rafe palatina, a anestesia local deve ser iniciada 
(CC BY 4.0) | ISSN 2525-3409 | DOI: http://dx.doi.org/10.33448/rsd-v9i10.8414

de anterior para posterior nos locais de perfurações, para assim durante as aplicações o líquido anestésico ir se dissipando para trás, fazendo com que apenas a primeira introdução da agulha seja percebida.

Em indivíduo que apresenta necessidade de protração maxilar e correções de assimétrias faciais, as corticoperfurações serão realizadas também nas cristas infrazigomáticas para assim facilitar a movimentação lateral dos hemiarcos.

Figuras 7 e 8. Anestesia da rafe palatina e crista infra zigomática.
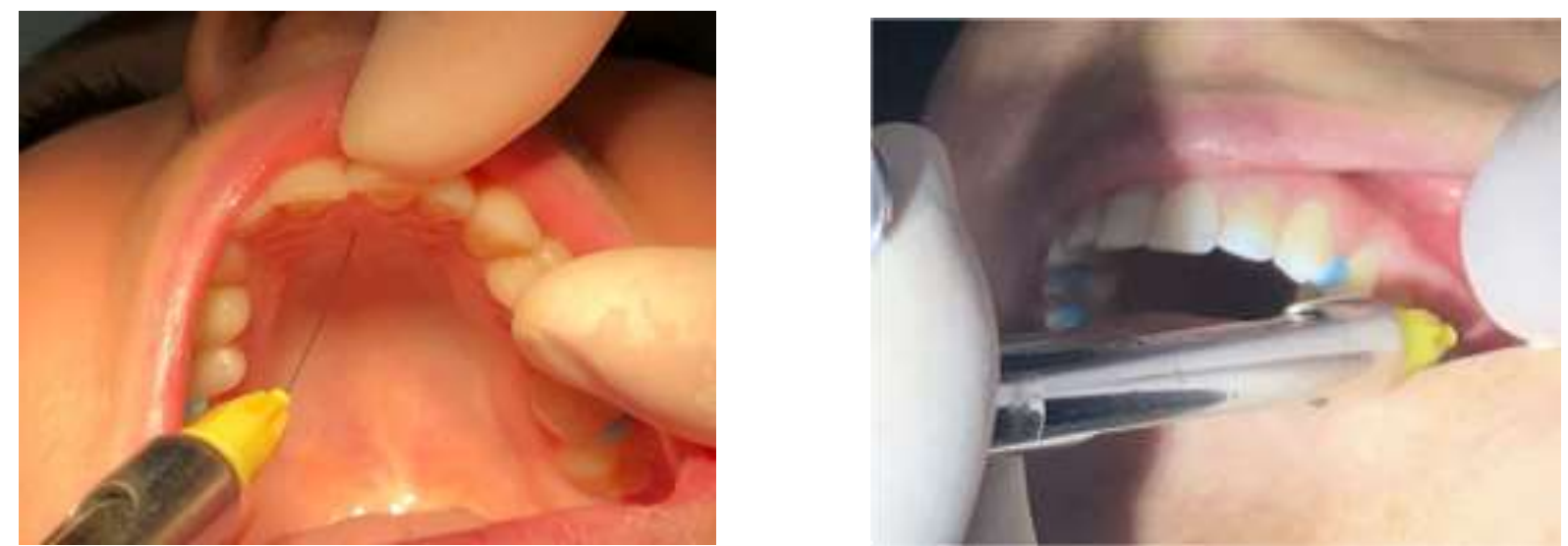

Fonte: O próprio autor.

Figuras 9 e 10. Pontos de perfurada na Rafe Palatina e da crista infra zigomática.
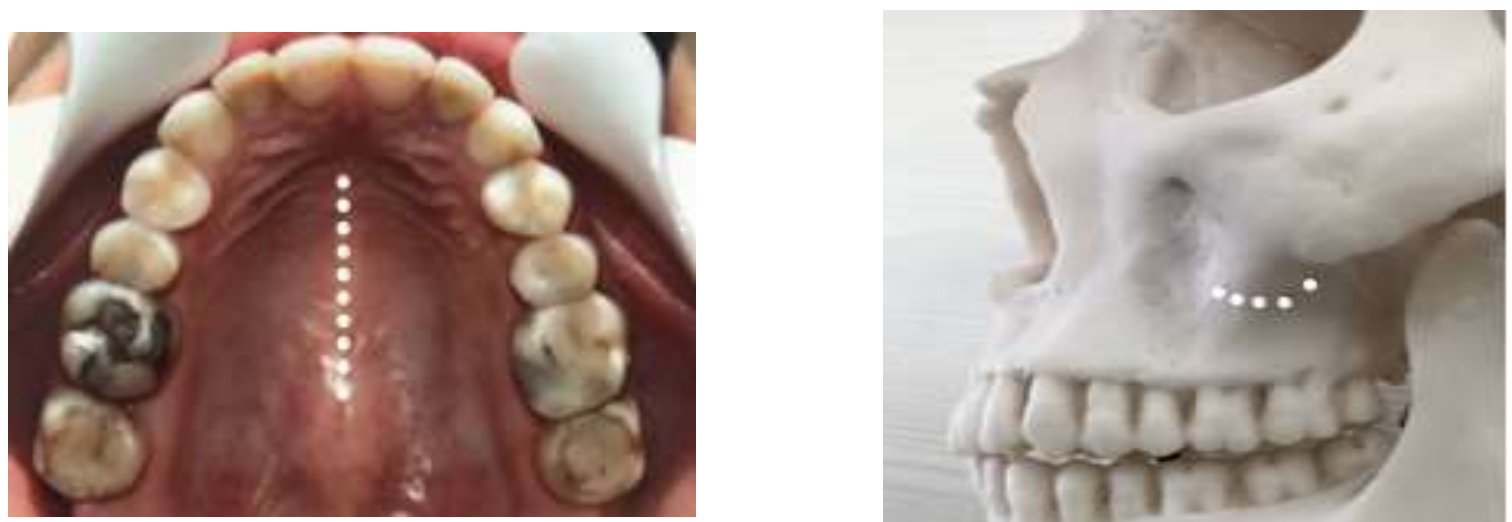

Fonte: O próprio autor.

Destaca-se que uma vez observado a presença de Torus palatino, concentra-se as perfurações nesta área de forma mais eficiente e mais próxima possível, garantindo que a fragilização desta área também aconteça. 
Figura 11. Kit completo de mini-implante intra e extra-alveolar e motor de redução.

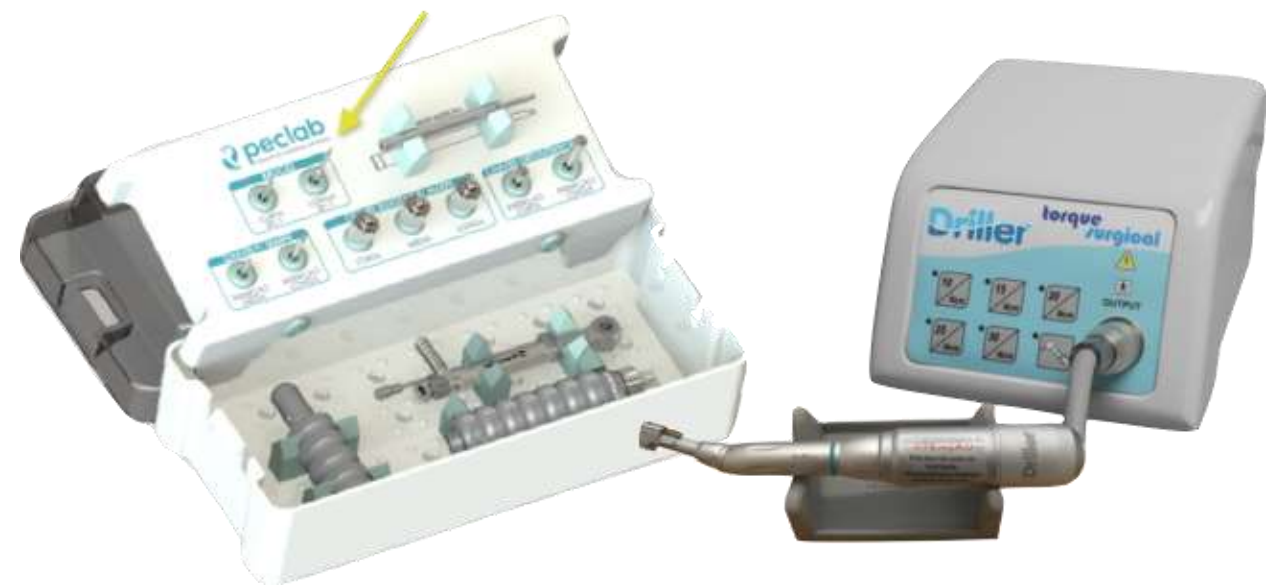

Fonte: https://peclab.com.br/educacao-conhecimento/ ; http://driller.com.br/

Os instrumentais utilizados para a fixação do parafuso expansor bem como para as perfurações, devem ser de boa qualidade e estarem devidamente esterilizados para que se evite possíveis contaminações e processos inflamatórios subsequentes.

Da mesma forma que a anestesia é aplicada, as perfurações ao longo da rafe devem ser realizadas utilizando-se uma broca específica para essa finalidade de forma que de milímetro em milímetro possa ser fragilizada a resistência do palato duro.

Figuras 12 e 13. Corticoperfurações da rafe palatina e sutura palatina mediana e seus três segmentos anterior, médio e posterior que se relacionam com outras suturas como, por exemplo, a palatina transversa e ossos nasais.
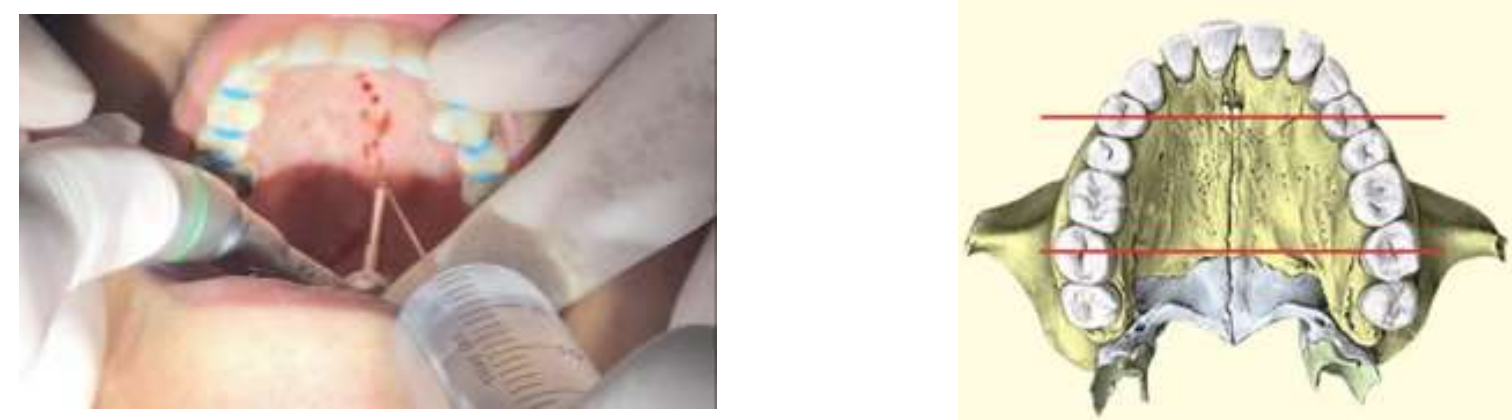

Fonte: O próprio autor. Fonte: Suzuki et al., Rev Clín Ortod Dental Press. (2016)

Os limites anteriores e posteriores do palato de maior resistência à expansão, devem ser perfurados para aumentarmos a eficiência da expansão pretendida. 
Figuras 14 e 15. Corticoperfurações realizadas na Rafe e anestesia nos locais de fixação dos parafusos
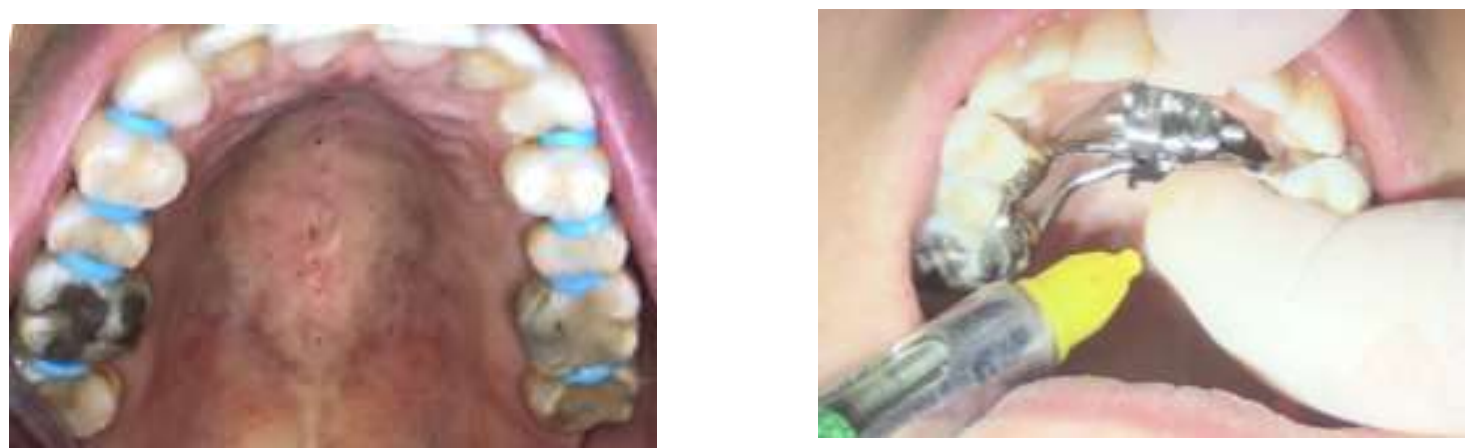

Fonte: O próprio autor.

Depois das perfurações realizadas marcas ao longo da rafe palatina ficam evidenciadas, mostrando onde a broca foi introduzida. Após a cimentação do parafuso expansor, novas anestesias precisam ser feitas nos locais onde serão introduzidos os parafusos de fixação.

Os parafusos devem ser fixados de forma cruzada, ou seja, primeiro instala-se um anterior da direita e, em seguida outro posterior da esquerda repedindo o procedimento com os outros parafusos. A checagem da estabilidade deve ser feita com uma chave de torquímetro, normalmente obtendo-se $30 \mathrm{~N}$ de aperto de cada parafuso.

Figuras 16 e 17. Instalação dos mini-implantes e utilização de torquímetro.
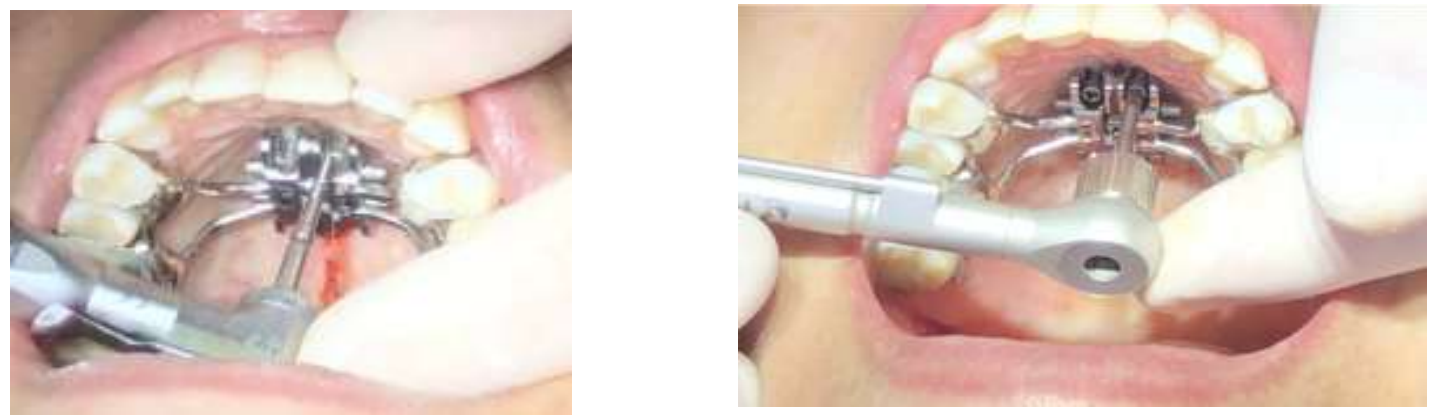

Fonte: O próprio autor.

Logo após a cimentação e fixação dos parafusos, as ativações podem ser iniciadas, variando conforme a resistência óssea encontrada no momento das corticoperfurações, bem como da idade do indivíduo e condições periodontais. Apesar dos protocolos encontrados na literatura, foi realizado $4 \times$ de $1 / 4$ de volta inicial para se obter 1 volta completa no parafuso, promovendo uma primeira impactacão de força sobre a rafe palatina e crista zigomática já fragilizadas pelas perfurações. Durante as ativações, além de demonstrado a forma correta de 
se fazer, foi também orientado para que nos dias seguintes fosse ativado, $2 \times$ de $1 / 4$ de volta por dia, sendo $1 / 4$ de volta de manhã e outra $1 / 4$ de volta a tarde, durante 7 dias. Haas (1970)

Após esse período, a paciente retornou para avaliações, nas quais foi observada a sobrecorreção da expansão necessária (vertentes internas das cúspides de trabalho ainda em contato, quase em topo a topo) e só então o parafuso foi travado. Se a sobrecorreção não tivesse sido alcançada, mais ativações seriam necessárias, devendo ser realizada até que se atinja o resultado esperado, com observações periódicas a cada 2 ou 3 dias subsequentes.

Figuras 18 e 19. MARPE instalado e logo após ativações - Uma perfeita adaptação deve ser conseguida sem áreas de isquemias mesmo depois das ativações realizadas.
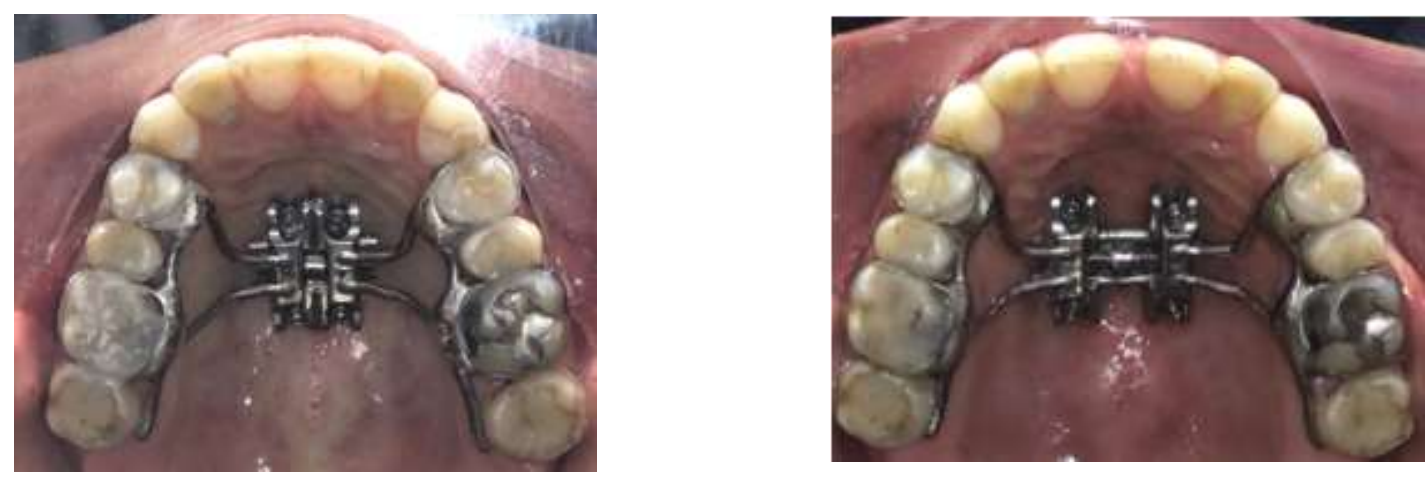

Fonte: O próprio autor.

Conseguida a expansão desejada, pode ser observado uma expansão maior na maxila do lado em que foram realizadas as perfurações da crista infrazigomática, bem como um deslocamento da mandíbula para o lado da expansão, diminuindo o desvio da linha média dentária e da assimetria facial.

Com a contenção do torno expansor depois de observado os ganhos transversais e a abertura do diastema anterior entre incisivos, é indicado a instalação de braquetes nos dentes incisivos centrais, laterais e caninos do lado do desvio e confecção de lace back entre eles (Fig. 21), lado onde a crista infrazigomática não foi perfurada, impedindo que esses dentes sejam tracionados pelas fibras supra-alveolares que fecham quase que totalmente o diastema aberto. Com isso, o deslocamento para a mesial se dará apenas nos dentes da hemimaxila expandida, fazendo com que o desvio de linha média dentária seja quase que totalmente corrigida. 
Figuras 20 e 21. Foto oclusal frontal inicial e foto oclusal frontal pós-expansão.
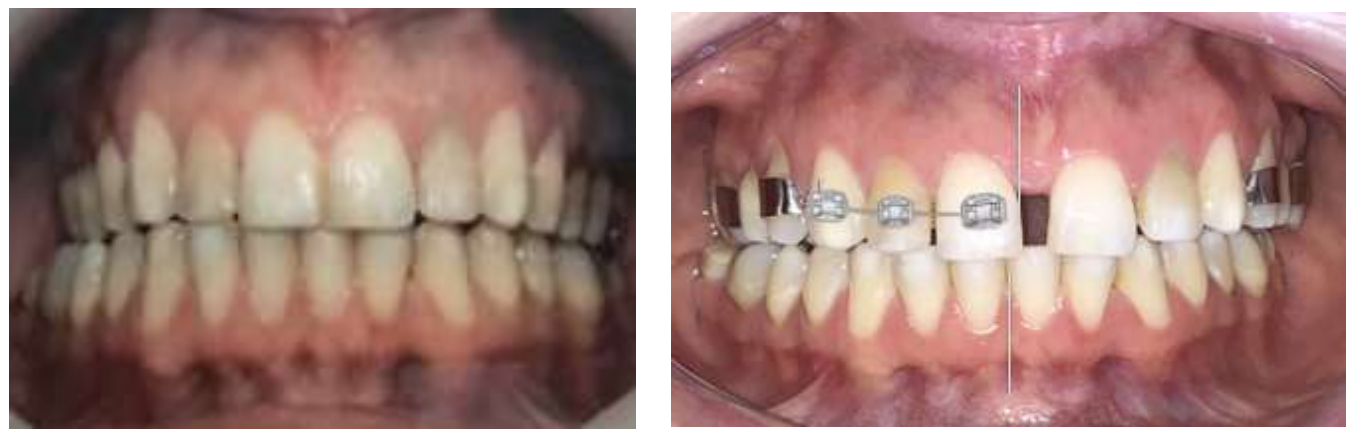

Fonte: O próprio autor.

O maior deslocamento da maxila pode ser observado tanto pela expansão conseguida do lado não cruzado, onde as perfurações foram realizadas quanto pela palpação na região da crista infrazigomática que se desloca para fora, quando comparado com o lado não perfurado anatomicamente preservado e que não sofre o deslocamento.

Figuras 22, 23 e 24. Foto lateral direita, foto oclusal e foto lateral esquerda.
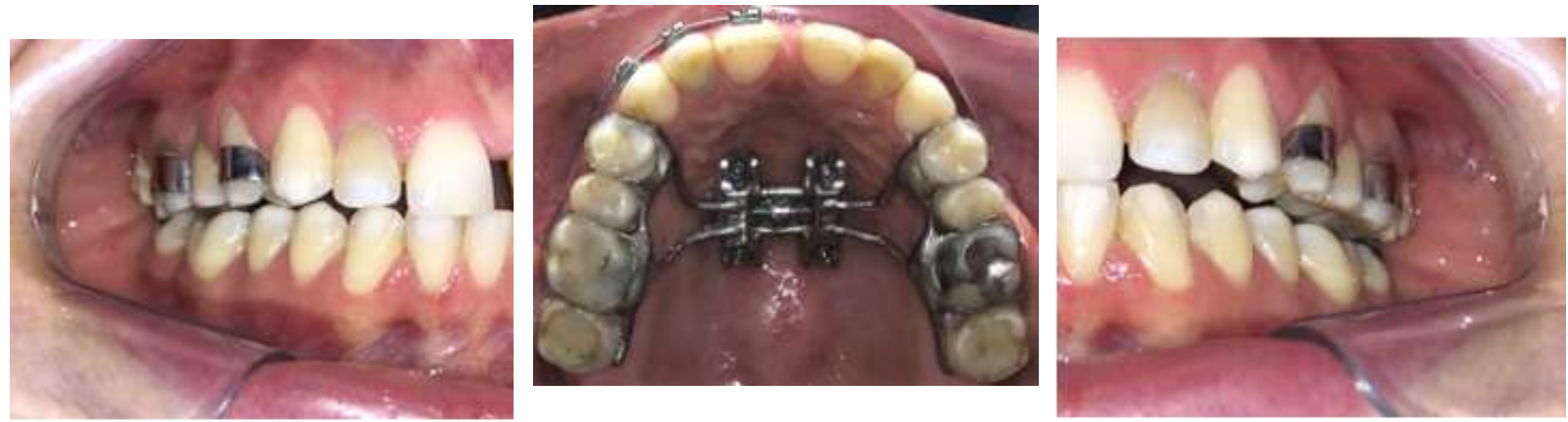

Fonte: O próprio autor.

Após a contenção do parafuso expansor deve-se aguardar um tempo médio de 3 meses para a reparação óssea da disjunção palatal Franchi et al. (2010), sendo indicado em muitos casos o corte com uma broca transmetal das hastes que saem do parafuso e vão em direção das bandas, removendo-se as partes do aparelho que não sejam o torno expansor fixado no palato Andrade (2018). Assim pode ser iniciada a instalação de todos os braquetes restantes para o tratamento ortodôntico, bem como iniciar o fechamento lento do diastema aberto entre os incisivos centrais, enquanto o torno expansor permanece fixado pelos parafusos, ancorando a expansão conseguida. 
(CC BY 4.0) | ISSN 2525-3409 | DOI: http://dx.doi.org/10.33448/rsd-v9i10.8414

Figuras 25 e 26. Foto oclusal pós-contenção e foto oclusal pós-remoção do MARPE.
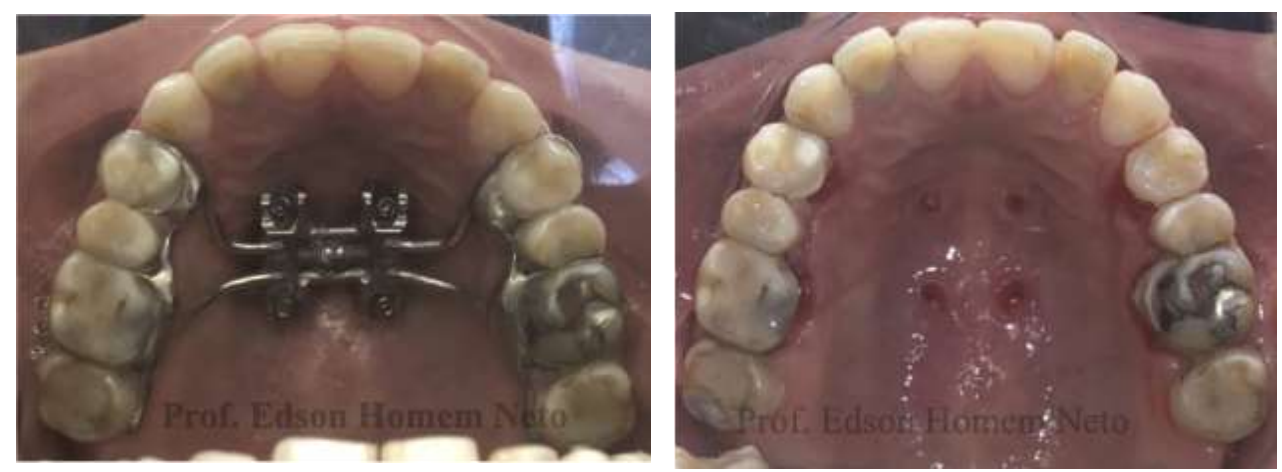

Fonte: O próprio autor.

Caso não seja deixado somente o torno expansor fixado no palato, e sim optado por deixar o conjunto durante o período de 3 meses de contenção, na remoção do aparelho (expansor e bandas), uma barra transpalatina normalmente é indicada para a manutenção do ganho transversal enquanto o tratamento corretivo da oclusão esteja sendo realizado. Haas (1970)

Figuras 27, 28 e 29. Aparelho instalado - lado direito, frontal e lado esquerdo.
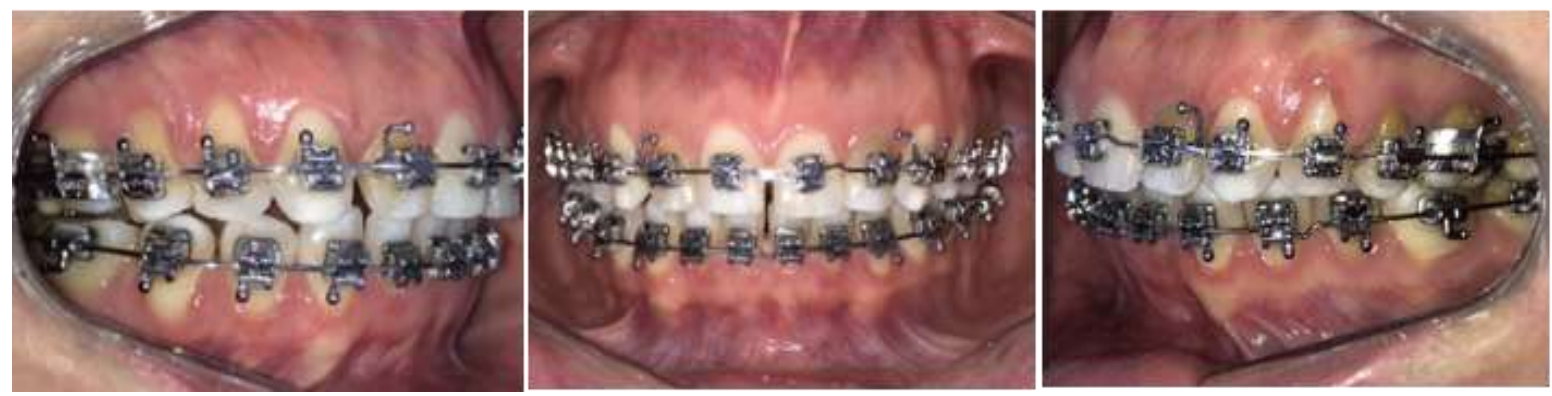

Fonte: O próprio autor.

Utilizando todos os princípios de uma mecânica autoligável Figueiredo, Pacheco \& Moura (2016); Maltagliati et al. (2013), o caso foi concluído em uma oclusão mutuamente protegida e com máxima intercuspidação habitual coincidente com relação centrica, decisivo no tratamento da disfunção da ATM apresentada no início. Alguns espaços foram deixados para o aumento dos dentes com proporções menores que as ideais (incisivos laterais superiores), aumentados após a remoção do aparelho. A linha média dentária e facial foi corrigida dentro da proposta oferecida inicialmente para a paciente. 
(CC BY 4.0) | ISSN 2525-3409 | DOI: http://dx.doi.org/10.33448/rsd-v9i10.8414

Figuras 30. 31 e 32. Fotos oclusais final, lateral direita, frontal e lateral esquerda.

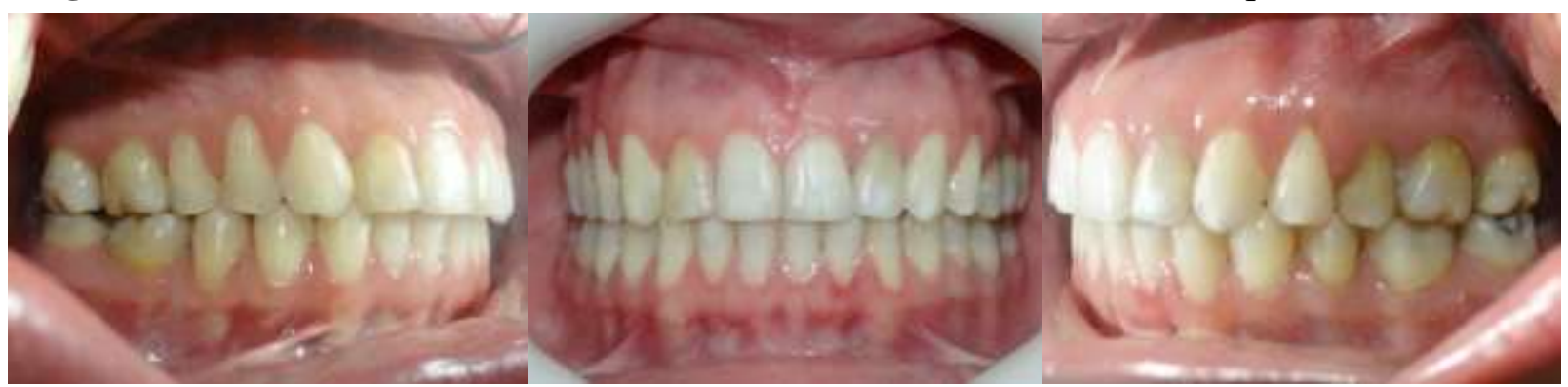

Fonte: O próprio autor.

Com a finalização do caso e remoção completa de todo o aparelho, um ajuste oclusal normalmente deve ser realizado para se obter um encaixe oclusal de melhor qualidade, visto que os desgastes naturais não aconteceram em harmonia com uma oclusão ideal.

Figuras 33, 34 e 35. Fotos frontais de sorriso, inicial, pós-expansão e final.
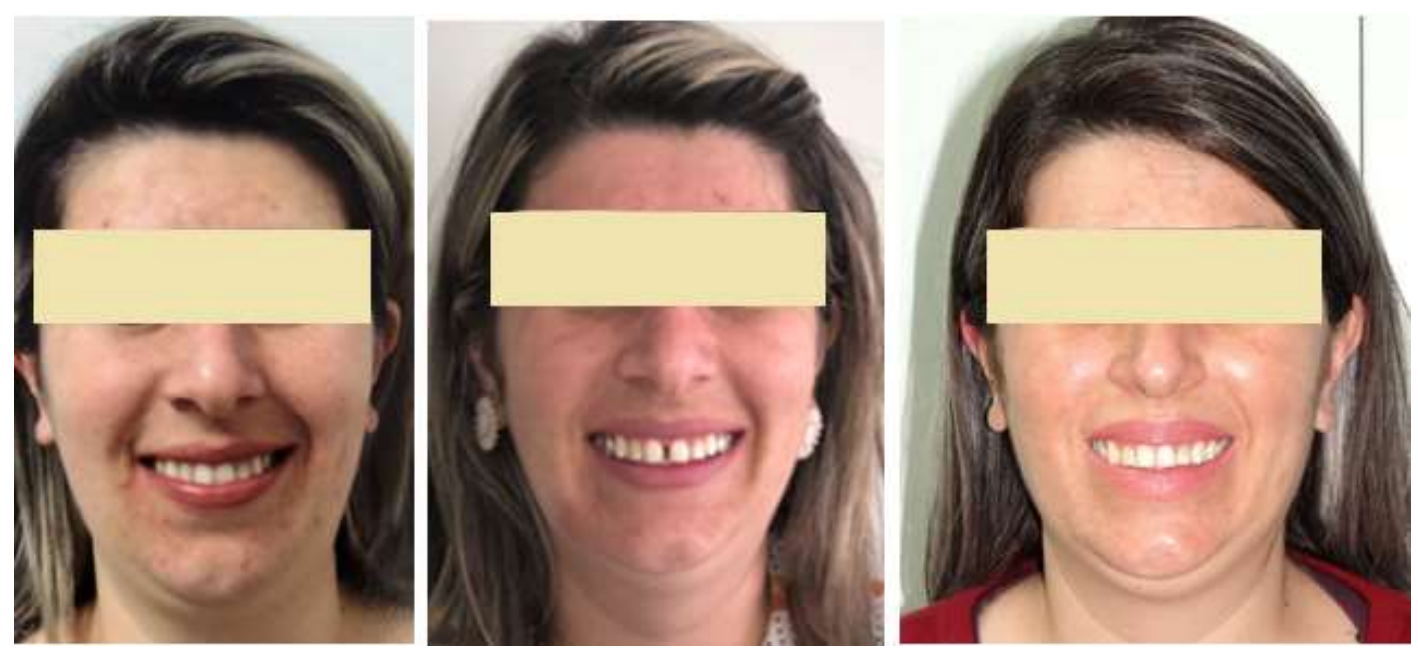

Fonte: O próprio autor.

As fotos frontais de sorriso comprovam a harmonia da face, bem como o deslocamento do mento para o lado esquerdo, obtido com as correções do tratamento. Pode-se notar a coincidência da linha média dentária com a linha sagital mediana da face.

\section{Discussão}

Avaliando-se os resultados obtidos nesta nova técnica de expansão com as perfurações das cristas infrazigomáticas em conjunto às realizadas na rafe palatina, pode-se observar que além de uma intervenção mais barata e rápida de ser feita, pode também ser 
observado os deslocamentos uni ou bilaterais nas regiões dos pilares zigomáticos, o que raramente é notado em relação as outras suturas que envolvem o complexo maxilar. Suzuki (2019)

Um outro fator observado que favorece a abordagem de expansão com corticoperfurações das cristas infrazigomáticas é o de minimizar as possíveis instabilidades dos miniparafusos, quando ativados independente do protocolo utilizado e as movimentações vestibulares dos dentes ancorados. Garib (2007)

Mesmo sabendo que as áreas de maior dissipação de força durante as ativações do expansor, continuam sendo a placa bucal dos dentes ancorados e do processo frontal do osso maxilar e não a área da sutura palatino Seong (2017), a melhor distribuição das forças com o uso dos parafusos de fixação do expansor e a menor resistência ao deslocamento lateral na região das cristas zigomáticas, diminuem a tensão sobre essas áreas o que normalmente causa certa preocupação.

As corticoperfurações realizadas com a intenção de fragilizar a abertura da sutura palatal, principalmente quando feitas na região de maiores resistências esqueléticas, além de facilitar o resultado pretendido requer menor quantidade de forças durante a mecânica de expansão Suzuki (2019) e na mesma linha de raciocínio são realizadas as corticoperfurações nas cristas infra zigomáticas.

Segundo Fonseca et al. (2015) existem três pilares verticais (nasomaxilar, zigomático e pterigomaxilar) do terço médio que fornecem o suporte primário nas direções verticais e anteroposterior da maxila. Esses pilares atuam dissipando as forças e transmitindo-as ao longo desses vetores, mantendo a posição espacial da maxila em relação ao crânio. Também de forma horizontal, existem pilares superiores, médios e inferiores que fornecem estabilidade lateral ao esqueleto facial sendo eles compostos pela lâmina orbital do osso frontal e cribriforme do etmoide; processo zigomático do osso temporal; corpo e processo temporal do zigoma; processo infraorbitário; superfície orbitária da maxila e segmento do processo frontal da maxila e; crista alveolar e palato duro que fornecem estabilidade lateral ao esqueleto facial central. Portanto, a fragilização das cristas infrazigomáticas seria mais um artifício para diminuir essas resistências anatomicamente existentes.

Baseado nesses princípios e pensando na diminuição da resistência a forças que possibilitem um novo rearranjo maxila, as corticoperfurações são fundamentais, principalmente quando se pretende um deslocamento lateral e ou unilateral para a correção de assimetrias, quando realizadas ao longo da rafe palatina e das cristas infrazigomáticas. 


\section{Considerações Finais}

As corticoperfurações de rafe palatina e de cristas infrazigomáticas, abrem um novo horizonte nas correções das assimetrias faciais e protrações maxilares, bem como nos problemas transversais da oclusão. Quando realizadas antes da expansão maxilar e em conjunto com os disjuntores apoiados em miniparafusos em indivíduos adultos, fragilizam as áreas de maior resistência para a obtenção do deslocamento lateral das estruturas maxilares, favorecendo as correções estéticas da face. O que antes parecia impossível de ser resolvido, sem as cirurgias ortognáticas, em alguns casos pode ser totalmente solucionado com essa nova metodologia.

Apesar dos resultados obtidos, novos trabalhos poderão comprovar a técnica descrita e acrescentar sugestões que possam melhorar os procedimentos, introduzindo como, por exemplo, um guia inicial de instalação e imagens de tomografias computadorizadas, comprovando os deslocamentos esqueléticos observados clinicamente.

\section{Referências}

Andrade, T. (2018). MARPE: uma alternativa não cirúrgica para o manejo ortopédico da maxila: parte 1. Rev Clín Ortod Dental Press. Out-Nov; 17(5), 44-55. 2.

Araújo, T. M. de, Nascimento, M. H. A., Bezerra, F., \& Sobral, M. C. (2006). Ancoragem esquelética em Ortodontia com miniimplantes. Revista Dental Press de Ortodontia e Ortopedia Facial, 11(4), 126-156. https://doi.org/10.1590/S1415-54192006000400014.

Arnett, G. W., Bergman, R. T., \& Barbara, S. (1915). 27. Facial keys to orthodontic planning. Part I diagnosis and treatment. American Journal of ORTHODONTICS and DENTOFACIAL ORTHOPEDICS Founded In, 103(4), 299-312.

Capelozza Filho, L., \& Silva Filho, O. G. da. (1997). Expansão rápida da maxila: considerações gerais e aplicação clínica. Parte I. Revista Dental Press de Ortodontia e Ortopedia Maxilar, 2, 88-102.

Franchi, L., Baccetti, T., Lione, R., Fanucci, E., \& Cozza, P. (2010). Modifications of midpalatal sutural density induced by rapid maxillary expansion: A low-dose computed- 
tomography evaluation. American Journal of Orthodontics and Dentofacial Orthopedics: Official Publication of the American Association of Orthodontists, Its Constituent Societies, and the American Board of Orthodontics, 137(4), 486-488; discussion 12A-13A. https://doi.org/10.1016/j.ajodo.2009.10.028.

Figueiredo, C., Pacheco, V., \& Moura, W. Ortodontia Autoligável e Contemporânea. (6a ed.), São Paulo: V8; 2016.

Fonseca, R. J., Walker, V., Dexter, B. H., Powers, M. P., Frost, D.E. Oral and Maxillofacial Trauma. (4a ed.), Editora Elsevier, 2015. 10.

Garib, D. G., Navarro, R. de L., Francischone, C. E., \& Oltramari, P. V. P. (2007). Expansão rápida da maxila ancorada em implantes: uma nova proposta para expansão ortopédica na dentadura permanente. Revista Dental Press de Ortodontia e Ortopedia Facial, 12(3), 75-81. https://doi.org/10.1590/S1415-54192007000300008.

Garib, D. G., Henriques, J. F., Janson, G., de Freitas, M. R., \& Fernandes, A. Y. (2006). Periodontal effects of rapid maxillary expansion with tooth-tissue-borne and tooth-borne expanders: a computed tomography evaluation. American journal of orthodontics and dentofacial orthopedics: official publication of the American Association of Orthodontists, its constituent societies, and the American Board of Orthodontics, 129(6), 749-758. https://doi.org/10.1016/j.ajodo.2006.02.021

Gurgel, J. A., Tiago, C. M., \& Normando, D. (2014). Transverse changes after surgically assisted rapid palatal expansion. International journal of oral and maxillofacial surgery, 43(3), 316-322. https://doi.org/10.1016/j.ijom.2013.10.001

Haas A. J. (1970). Palatal expansion: just the beginning of dentofacial orthopedics. American journal of orthodontics, 57(3), 219-255. https://doi.org/10.1016/0002-9416(70)90241-1.

Lee, K. J., Park, Y. C., Park, J. Y., \& Hwang, W. S. (2010). Miniscrew-assisted nonsurgical palatal expansion before orthognathic surgery for a patient with severe mandibular prognathism. American journal of orthodontics and dentofacial orthopedics: official publication of the American Association of Orthodontists, its constituent societies, and the 
Research, Society and Development, v. 9, n. 10, e7589108414, 2020

(CC BY 4.0) | ISSN 2525-3409 | DOI: http://dx.doi.org/10.33448/rsd-v9i10.8414

American

Board

of

Orthodontics, 137(6),

$830-839$.

https://doi.org/10.1016/j.ajodo.2007.10.065

Maltagliati, L. A., Myiahira, Y. I., Fattori, L., Filho, L. C., \& Cardoso, M. (2013). Transversal changes in dental arches from non-extraction treatment with self ligating brackets. Dental press journal of orthodontics, 18(3), 39-45. https://doi.org/10.1590/s217694512013000300008 .

Murata, W. H., Oliveira, C. B., Suzuki, S. S., Suzuki, H. Expansão Rápida da Maxila Assistida por Mini Implantes Ortodônticos. In: Ortodontia - Estado Atual da Arte: Diagnóstico, Planejamento e Tratamento, Ed. Napoleão, 2017, 311-333.

Pereira, A. S., Shitsuka, Dorlivete Moreira Parreira, F. J., \& Shitsuka, R. (2018). Metodologia da Pesquisa Científica - Licenciatura em Computação. Retrieved from https://repositorio.ufsm.br/bitstream/handle/1/15824/Lic_Computacao_MetodologiaPesquisa-Cientifica.pdf? sequence $=1$.

Perin, M. A. A. (2016). Expansão Rápida Da Maxila Assistida Por Mini-Implantes (Marpe) Em Pacientes Adultos, 28. https://acervodigital.ufpr.br/bitstream/handle/1884/53914/R\%20$\%$ 20E\%20\%20MARIA\%20AUGUSTA\%20ANDRIGO\%20PERIN.pdf?sequence=1\&isAllo wed=yMonografias 2016.

Remmelink H. J. (1988). Orientation of maxillary sutural surfaces. European journal of orthodontics, 10(3), 223-226. https://doi.org/10.1093/ejo/10.3.223

Santos, A. M., et al. Expansão rápida da maxila assistida com mini-implantes (MARPE) em adulto. In: Dental Press Publishing / Rev Clin Ortod Dental Press / Out-Nov, 18(5),110-7

Santos, A. M., et al. Protocolo de expansão rápida da maxila assistida por mini-implantes (Marpe) associado à corticotomia. In:Ortodontia SPO / trabalho original. 2019; 51(1), 42-8.

Seong, E.-H., Choi, S.-H., Kim, H.-J., Yu, H.-S., Park, Y.-C., \& Lee, K.-J. (2018). Evaluation of the effects of miniscrew incorporation in palatal expanders for young adults using finite 
element analysis. The Korean Journal of Orthodontics, 48(2), 81. https://doi.org/10.4041/kjod.2018.48.2.81

Simões, W. Visão do crescimento mandibular e maxilar. J. Bras. Ortodon. Ortop. Facial; 3(15):9-18, 1998.

Suzuki, H., Moon, W., Previdente, L. H., Suzuki, S. S., Garcez, A. S., \& Consolaro, A. (2016). Expansão rápida da maxila assistida com mini-implantes MARPE: em busca de um movimento ortopédico puro. Revista Clínica de Ortodontia Dental Press, 15( 2), 100-108.

\section{Porcentagem de contribuição do autor no manuscrito}

Edson Homem Neto - 100\% 